\title{
Der ironische Modus im aktuellen Liebesroman
}

\section{The Ironical Mode in the Contemporary Romance Novel}

\begin{abstract}
The ironical mode is a complex of literary, especially structural, metafictional, intertextual, distancing, and illusion-breaking methods that are used in contemporary German romance novels. A relativistic notion of love as cultural construct underlies this formal organization. In the paper the ironical mode is opposed with the utopian mode, and questions concerning its efficacy in the contemporary German literature are posed. Romance novels by Wolf Haas, Monique Schwitter, Christoph Höhtker, Peter Handke, Martin Walser, Friedrich Christian Delius, Navid Kermani, Brigitte Kronauer, Olga Flor, Elfriede Jelinek, Sibylle Berg, Marlene Streeruwitz and Corinna T. Sievers are approached.

Keywords: romance novel, love, irony, contemporary German literature.
\end{abstract}

Den gängigen Thesen von Enttarnung und Abwertung des Mythos der romantischen Liebe in der Postmoderne lässt sich ein unschwer bemerkbares Paradox entgegenhalten, dass der besagte Mythos eben von genuin postmodernen Medien unterstützt, verbreitet, intensiviert und verkauft wird. Die Allgegenwart der Liebe idealistisch-romantischer (utopischer) Prägung in audiovisueller Werbung, sozialen Netzwerken, Musikhits, Filmschlagern und Literatur bestreitet einerseits den antiutopischen Charakter der Postmoderne als Kulturformation, andererseits bestätigt dies den wahrscheinlich längst akzeptierten Verdacht, dass die „Epoche“ der Postmoderne zahlreiche unzusammenhängende oder widersprüchliche Tendenzen umfasst und sich als Zeit der maximalen Pluralisierung von Ideen und Diskursen auffassen lässt. 
Der Großteil der aktuellen Kulturproduktion scheint die Konstruiertheit der romantischen Liebe mit dem Glauben an ihre Natürlichkeit zu verschleiern, mehr noch: die ihm zugrundeliegende Materialität, soziale und ökonomische Verhältnisse zu leugnen: „An ihre Stelle ist ein diskreter und ätherischer Reichtum getreten, der sich auf subtile Weise an ,universelle' und klassenlose Sehnsüchte nach Authentizität, Freiheit und reinem Gefühl richtet." Freilich ist diese Diagnose selbst wenig universell - die Aufdeckung des in unzähligen Kulturtexten inszenierten Theaters der Liebe hängt von sozialen Dispositionen ab, in erster Reihe von Bildung und Belesenheit, so ist auch die Teilnahme an einer bestimmten „Rezeptionskultur der Liebe“ eine relative Frage. Die an Sicherheit grenzende Vermutung, dass viele Rezipienten die romantische Illusion kritisch zu durchschauen imstande sind und trotzdem in diesem medial kreierten Liebestheater mitspielen möchten, bestätigen nicht nur soziologische Forschungen, wie etwa die von Eva Illouz, die diesem Paradox von Erkenntnis und Teilnahme nachspürt, ${ }^{2}$ sondern auch die verbreiteten, normalerweise westlichen oder globalisierten (amerikanisierten), Rezeptionsmechanismen von solchen Produkten der Unterhaltungsindustrie wie z. B. Fifty Shades of Grey (Roman und Verfilmung), die sich sowohl in einer (auto)ironischen, zweifelnden Haltung als auch im übermäßigen Konsum und der darauffolgenden Re-Produktion von Phantasmen (hauptsächlich Überarbeitung eigener Erfahrungen nach dem mehr oder weniger bewusst angenommenen medialen Muster) offenbaren. Die Kraft dieser als Lebensszenarien gelesenen Simulakren wirft nicht nur die Frage nach den aktuellen Veränderungstrends der Gesellschaft auf, ${ }^{3}$ sondern auch nach der Beständigkeit von Kulturschemata, die den Konsum der Romantik bedingen, sowie auch nach der Wirksamkeit der rhetorischen und formalen Mittel, die bei deren Produktion und Rezeption eingesetzt werden.

Der utopische Modus des „Liebemachens“ (verstanden hier als Konstruktion von persuasiven Liebeserzählungen), ${ }^{4}$ der im Folgenden kurz besprochen wird, lässt sich in diversen Medien und Genres entdecken, hier wird er nur in Bezug auf den aktuellen, hauptsächlich deutschsprachigen Liebesroman und als Kontrastfolie zum weiter dargestellten ironischen Mo-

1 Eva Illouz, Der Konsum der Romantik. Liebe und die kulturellen Widersprüche des Kapitalismus, übers. v. Andreas Wirthensohn (Frankfurt am Main: Suhrkamp, 2007), 138-139.

2 Vgl. Illouz, Der Konsum der Romantik. Liebe und die kulturellen Widersprüche des Kapitalismus, 136-137.

3 Vgl. Niklas Luhmann, Liebe als Passion. Zur Codierung von Intimität (Frankfurt am Main: Suhrkamp, 2015), 24.

4 Analog zum in der Genderforschung angewandten Terminus doing masculinity/femininity, der auf die Konstruiertheit der sozialen Geschlechter hinweist. 
dus identifiziert. ${ }^{5}$ Gemeint ist hier ein Komplex von formalen und meistens konventionellen literarischen Verfahren, dem ebenso ein Komplex von geregelten und wenig flexiblen kulturellen Überzeugungen zugrunde liegt.

Auf der Inhalts- bzw. Ideenebene des Textes handelt es sich in erster Linie um die Überzeugung von der Existenz des unveränderlichen Selbst und von der Natürlichkeit menschlichen Verhaltens, nicht selten auch von der unbestreitbaren Gegebenheit gesellschaftlicher Strukturen und Geschlechterverhältnisse, sowie um den Glauben an den universellen Ursprung und Wert der Liebe. Der utopische Modus ist somit ahistorisch. Weitere Implikationen dieses Liebeskonzepts lassen sich wie folgt zusammenfassen: Der Ursprung der Liebe ist entweder die unabhängige Gefühlswelt des Menschen oder eine übermenschliche Instanz, die Liebe wird gesucht und gefunden (oft nach dem herkömmlichen, „platonischen“ Muster der Wiedervereinigung), bildet entweder einen Weg zur Selbstverwirklichung oder zur Verwandlung und Überschreitung des Ichs, ${ }^{6}$ nimmt sakrale, übernatürliche oder mystische Züge an. Der Moment der Verliebtheit krönt den Prozess des Wartens auf den Anderen (er beginnt die Liebesgeschichte im Grunde genommen nicht), die Liebe wird als ewig, einzigartig und höchster Lebenswert kodiert, wodurch sie alle anderen Handlungen im sozialen Umfeld rechtfertigt. Der utopische Modus setzt aber nicht unbedingt einen glücklichen Ausgang der Geschichte voraus.

Im utopischen Modus gibt es kaum kritische Reflexion in Bezug auf die „Natur" der Liebe. Auch wenn gegebene Bedingtheiten des Liebens hinterfragt werden können, werden sie nicht als dessen Essenz, sondern als unbeständige Staffage des unveränderlichen Gefühls angesehen. Diese Auffassung wird von idealistischen Philosophien und vielen Religionen unterstützt, ihre Wirksamkeit im europäischen Kulturkreis lässt sich auch auf zweierlei Weise erklären - mit dem Einfluss und der Aktualität des antiken (idealistischen, nicht sophistischen) Erbes sowie mit der führenden Rolle des Christentums in der Kulturbildung seit dem Mittelalter. Trotz der Diagnosen vom Ende der Liebe in der neoliberalen, medialisierten Leistungskul-

5 Der Terminus „Modus des Schreibens“ wird von Renate Lachmann in Bezug auf die Fantastik verwendet, vgl. Erzählte Phantastik. Zu Phantasiegeschichte und Semantik phantastischer Texte (Frankfurt am Main: Suhrkamp, 2002), 12.

6 Entgegen der popkulturellen Anbetung der „platonischen“ Liebe als harmonische, geistige Vereinigung sieht Byung-Chul Han die Liebe vom Altertum bis heute als Selbstüberschreitung, Verwandlung und Verletzung an: „Die erotische Kommunikation der Antike ist alles andere als behaglich“. Byung-Chul Han, Agonie des Eros (Matthes \& Seitz, Berlin, 2017), 42. Davon geht auch seine Kritik der gegenwärtigen Liebeskultur aus, die das ursprünglich negative, auf den Anderen ausgerichtete Gefühl domestiziert und ins Positive verarbeitet hat. Auf dieser Grundlage könnte auch eine weitere Einteilung des utopischen Modus in das negative und positive Muster erfolgen. 
tur der Gegenwart ${ }^{7}$ sind diese essentialistischen Überzeugungen erstaunlich erfolgreich und weit verbreitet, vor allem in der Digitalkultur, die sie gerne vervielfältigt.

Auf der Formebene spiegeln sich diese Annahmen meistens in der Wahl bestimmter Verfahren, allen voran einer linearen Struktur der Liebesgeschichte mit ihren obligatorischen Stationen, die möglichst „lebensnah“ wirken und die temporale Struktur der Liebessemantik widerspiegeln sollte. ${ }^{8}$ Bevorzugt werden mimetischer Realismus und tiefgründige Psychologisierung der Figuren, Glaubwürdigkeit und Erkennbarkeit der Handlungsmuster. Es kann somit nicht verwundern, dass im utopischen Modus meistens konventionalisierte Texte geschrieben werden, die nicht nur als Varietäten des ewig Gleichen wiedererkannt und gelesen werden, sondern auch die herkömmlichen Konventionen verfestigen und den Boden für nächste Narrationen bereiten.

Auf der Ebene der Rezeption äußert sich der utopische Modus vor allem in einer Identifikationslektüre, die in erster Linie die Lesenden mit den Protagonist(inn)en des Romans gleichsetzt, aber auch zu weiteren Identifikationen führen kann, beispielsweise zwischen Autor(in) und Erzähler(in) und/oder Protagonist(in), nicht selten auch zwischen Leser(in) und Autor(in). Diese Lektüre ist meistens auch stark prointentional, hier wird der literarische Text für die direkte Äußerung seines Urhebers gehalten und die in ihm kodierten Weltanschauungen als Stellungnahmen oder illokutive Sprechakte gelesen. Die verbreitete Konvention, Liebesromane wie Ratgeber oder Autobiografien zu lesen, findet ihren Ursprung eben in dieser (oft unbegründet vorausgesetzten) „Vertraulichkeit“ der literarischen Kommunikation. Dem ist auch ein Mangel an Kritik geschuldet, was jedoch nicht bedeutet, dass die im utopischen Modus geschriebenen Texte immer positiv gewertet werden, vielmehr handelt es sich hier um eine Kritik an der Oberfläche, z. B. Handlungswahrscheinlichkeit, Profilierung der Figuren, Spannungserzeugung oder sprachliches Niveau, seltener aber um die basalen Konzepte des Werks, wie die Suche nach Liebe und den Glauben an ihre ideale Natur. Derartige Lesarten beruhen in der Regel auf einem ethischen Konsens und die Disponierung der Werte scheint im Voraus bestimmt, daher bringt die Lektüre wenige Überraschungen oder moralische Dilemmata mit sich.

Die Frage nach der Geschichte des utopischen Modus ist insoweit riskant, als dieser erst durch die Opposition zu anderen Modi zum Vorschein kommt

7 Vgl. u. a. Han, Agonie des Eros (2012), Illouz, Warum Liebe endet (2018).

8 Vgl. Takemitsu Morikawa, „Gibt es ein Weltfunktionssystem für Intimbeziehungen? Jenseits des Spannungsverhältnisses von Kulturalismus und Westzentrismus“, in Liebesgeschichte(n). Identität und Diversität vom 18. bis zum 21. Jahrhundert, hrsg. v. Frank Becker und Elke Reinhardt-Becker (Frankfurt am Main: Campus, 2019), 341. 
und seine Existenz (mangels Alternative) nicht unbedingt immer und überall gleichmäßig bewusst gemacht wird. So könnte etwa der utopische Charakter der höfischen Dichtung des Mittelalters, der Literatur der Empfindsamkeit oder der Romantik im synchronen Vergleich mit anderen, nicht kanonischen Modi dieser Perioden, zum Beispiel in der Volkskultur, oder aber mit den gegenwärtigen Kulturen der Liebe und ihren Schreibmodi in Erscheinung treten. Erst in der Zeit der Modernisierung sowie der darauffolgenden Demokratisierung und Pluralisierung der Literatur wurden diese Oppositionen hervorgehoben, ja denkbar gemacht. Auf diese Weise konnten auch andere, bisher periphere oder tabuisierte Modi erscheinen und für gewisse Verbreitung sorgen. So behauptet Lynne Pearce, dass erst die Etablierung der neuen Wissenschaften in der Moderne eine andere, dystopische Liebesliteratur ermöglicht habe ${ }^{9}$ (die teilweise mit dem ironischen Modus gleichzusetzen ist). Noch allgemeiner könnte festgestellt werden, dass die Entwicklung der materialistischen Tendenzen in der Aufklärung, der modernen Soziologie und der relativistischen Denkströmungen in der Postmoderne die Grundlage für eine andere, nicht-idealistische Auffassung der Liebe gebildet haben.

Der utopische Modus wirkt quer durch Genre- und Texthierarchien, umfasst sowohl kanonische Werke der Liebesliteratur als auch zur Unterhaltungsliteratur eingeordnete Narrationen, die der so genannten romantischen Liebe huldigen. Auch ein Großteil der zurzeit populären erotischen Romane, die nach dem bereits erwähnten Erfolg von E. L. James' Shades of Grey (2011-2012) auftauchten und als hedonistisch oder pornografisch abgestempelt wurden, bekennt sich zur Utopie und behandelt den Sex als einen Weg zur emotionellen Verwirklichung und Stabilisierung, ja zur Entdeckung der „wahren Liebe“. Ähnlich verhält es sich mit vielen romantischen Komödien, die unter dem Deckmantel der Komik idealistischen Liebesprojekten verpflichtet sind. Die Liebesutopie wird auch - worauf Pearce hinweist stellenweise in der Science-Fiction, der Fantasy, dem Thriller und in der queeren Literatur glaubhaft gemacht, sowie im metafiktionalen Schreiben auf der ersten Erzählebene, worauf hier später eingegangen wird. ${ }^{10} \mathrm{Zu}$ den anerkannten gegenwärtigen Autor(inn)en des utopischen Modus in deutscher Sprache könnten unter anderem Peter Handke, Navid Kermani, Annette Mingels, Anna Mitgutsch, Hanns-Josef Ortheil, Evelyn Schlag, Peter Stamm, Martin Walser oder Feridun Zaimoglu gerechnet werden, mit dem Vorbehalt, dass sie, weit von der popkulturellen Affirmation der romantischen Liebe, durchaus innovative Darstellungsmittel verwenden und die Liebesutopie zeit-

9 Vgl. Lynne Pearce, Romance Writing (Cambridge: Polity Press, 2007), 162-164.

10 Vgl. Pearce, Romance Writing, 185-188. 
gemäß kontextualisieren. Manche Romane der genannten Autor(inn)en werden im Folgenden auch auf ihr ironisches Potenzial hin geprüft.

Der ironische Modus fußt auf der Annahme, dass Liebe - wie alle sozialen Phänomene - ein Konstrukt oder eine Erzählung ist und in eindeutig artifiziellen Formen ausgedrückt werden sollte. Reflektiert werden hier Konventionen des Liebens, Theatralität und Wiederholbarkeit der Liebesrituale, Möglichkeiten und Begrenzungen der Liebessprache, der Mythos Liebe angesichts des aktuellen Kulturwandels, der Einfluss von Liberalismus, Optimierung, Digitalisierung, Kommerzialisierung und Optionalität der Beziehungen. Meistens ist der ironische Modus kulturkritisch und antiutopisch. ${ }^{11}$ Er kann auch in verschiedenen Texten ungleichmäßig vorkommen und Momente einer kritischen Reflexion konstituieren, muss aber nicht unbedingt intentional aufgefasst werden, vielmehr als ein durch bestimmte kulturelle Erkenntnisse fundierter, mehr oder weniger sichtbarer Diskurs, der an der Oberfläche des Textes (vor allem in der sprachlichen Gestaltung) oder tiefer in seiner Struktur verläuft.

Eva Illouz sieht in der Ironie die tonangebende rhetorische Figur des heutigen romantischen Bewusstseins und desillusionierten Wissens, „das eine volle Überzeugung und Verbindlichkeit verhindert.“12 Und weiter: „Die Ironie kann einen Glauben nicht ernst nehmen, der für die Liebe zentral ist, nämlich ihren selbsterklärten Anspruch auf Ewigkeit und Totalität." ${ }^{13}$ Diese Desillusionierung ergibt sich aus dem Bewusstsein der Endlichkeit der Liebe, was jedoch - wie Illouz bemerkt - bereits in der Romantik (Schlegel) akzeptiert wurde, sowie aus der Rationalisierung der Liebe im Kapitalismus, welche sie der utopischen Züge beraubt hat. Immer häufiger auftretende Praktiken der Beziehungsoptimierung oder aber - ganz im Gegenteil - der alle anderen Lebenspraktiken optimierenden Liebesmeidung zeugen tatsächlich von einem erhöhten Bewusstsein der Gefahr, welche die romantische, transgressive Liebe mit sich bringt.

Hier stellt sich jedoch die Frage, in welchem Maße dieses ironische Bewusstsein sich in Produktion und Rezeption von Kulturartefakten widerspiegelt, mit anderen Worten: inwieweit die beiden sozialen Strukturen - die Struktur der Liebeskultur und die Struktur des literarischen Feldes (verstanden im Sinne von Pierre Bourdieu) ${ }^{14}$ - eine Homologie aufweisen. Zwischen

11 Zur Ironie im relativistischen Verständnis vgl. Richard Rorty, Contingency, irony, and solidarity (Cambridge: Cambridge University Press, 1993), 73-95, https://doi.org/10.1017/ CB09780511804397.

12 Eva Illouz, Warum Liebe weh tut. Eine soziologische Erklärung, übers. v. Michael Adrian (Berlin: Suhrkamp, 2011), 351.

13 Illouz, Warum Liebe weh tut. Eine soziologische Erklärung, 351.

14 Vgl. Pierre Bourdieu, Die Regeln der Kunst. Genese und Struktur des literarischen Feldes, übers. v. Bernd Schwibs, und Achim Russer (Frankfurt am Main: Suhrkamp, 1999), 228-229. 
dem ästhetischen (Avantgarde) und dem sozialen (Massenproduktion) Pol des literarischen Feldes, also zwischen der langen (ästhetischen) und der kurzen (sozialen) Wirkungszeit, ${ }^{15}$ scheint das ironische Liebesbewusstsein ungleichmäßig verteilt zu sein. Die Avantgarde und der Nobilitierungssektor ${ }^{16}$ sind in der Regel tatsächlich an der Desillusionierung der bestehenden Verhältnisse sensu largo beteiligt, weswegen der ironische Modus ein wesentliches Verfahren in Liebesromanen dieser Formationen bildet. Dabei sind der Mittelbereich und - weiter in Richtung soziale Zeit - das Feld der literarischen Massenproduktion immer weniger an der Aufhebung der Mythen interessiert, die der Populärkultur zugrunde liegen. Ihre Ziele sind vor allem Unterhaltung und Kompensation, sie profitieren wenig von Widerstand und Desillusionierung, da die massenweisen, attraktiven und ihre Konsumenten abhängig machenden Illusionen eben aufrechterhalten bleiben müssen, um sich verkaufen zu lassen. Wenn die Ironie hier wirken sollte, dann in einer schwachen Form, wie etwa in der populären chick-lit und verwandten Genres, die tatsächlich die Unbeständigkeit der Liebe, sexuelle Freiheit, Konsum und Sozialprestige besingen, ohne zur weitergehenden Reflexion zu zwingen oder an den Fundamenten der vorgefundenen Ordnung zu rütteln. ${ }^{17}$

Einerseits wird hier also der utopische Liebesmythos in seiner naivsten Gestalt reproduziert, andererseits die Sexualität zur Schau gestellt und als angenehme Unterhaltung stilisiert. Die Rezeption derart profilierter, widersprüchlicher Trends und Artefakte muss in manchen Fällen Unsicherheit oder Distanz generieren, deren künstlerische Form eben der ironische Modus mit seinem Bestreben, die Dialektik von Liebe und Körper außer Gefecht zu setzen, bildet. Der ironische, nicht der lediglich komische, sarkastische oder zynische, ${ }^{18}$ sondern der radikal umkehrende und selbstreflexive Modus der Liebesliteratur ist von einem kritischen Bewusstsein gekennzeichnet, das

15 Vgl. Heribert Tommek, Der lange Weg in die Gegenwartsliteratur. Studien zur Geschichte des literarischen Feldes in Deutschland von 1960 bis 2000 (Berlin/New York: De Gruyter, 2015), 581.

16 Terminologie Tommeks, vgl. Tommek, Der lange Weg in die Gegenwartsliteratur. Studien zur Geschichte des literarischen Feldes in Deutschland von 1960 bis 2000.

17 Auch wenn die chick-lit strenggenommen keine Liebesliteratur sein sollte, vgl. Pearce, Romance Writing, 182-183. Vgl. auch den Modus des „kalten“, unverbindlichen und sachlichen Erzählens von Liebe und Sexualität, vgl. Monika Szczepaniak, „Coole Romanze als literarisches Programm“, in Liebesgeschichte(n). Identität und Diversität vom 18. bis zum 21. Jahrhundert, hrsg. von Frank Becker, und Elke Reinhardt-Becker (Frankfurt am Main: Campus, 2019), 151-152.

18 Pierre Lepape behauptet sogar, dass es keinen komischen Liebesroman gebe, da die Liebenden sehr selten komisch sind, vgl. Pierre Lepape, Une histoire des romans d'amour (Paris: Seuil, 2011), 16. 
sich gegen jegliche, sowohl idealistische als auch materialistische, Mystifizierung wendet. Auf der Formebene äußert sich diese diskursive Prägung in der Wahl von unkonventionellen Mitteln, vor allem hybriden, verfremdeten Formen, nichtlinearen Handlungen, Verfahren der Metafiktionalität und Intertextualität, Illusionsbrechung oder umgekehrt - übertriebener Theatralisierung. Die Sprache des ironischen Modus ist auch nicht selten metafiktional, bewusst ihrer selbst und ihrer Funktion in der Konstruktion von Liebesgeschichten. Die Protagonist(inn)en können als Rollen oder Marionetten dargestellt werden bzw. ihre eigene Erfahrung und ihr Darstellungsvermögen hinterfragen. Auf dem Weg zur kritischen Erkenntnis bildet die Ablehnung des direkt wirkenden Realismus den ersten Schritt, wozu sich metafiktionale Verfahren eignen, aber auch weniger offensichtliche Signale, wie zum Beispiel der ironische oder unbeteiligte Ton des Erzählers oder auch die Künstlichkeit der Dialoge (die an sich doch immer eine theatrale Form sind).

Die obigen formalen Lösungen bzw. genrespezifischen Verfremdungseffekte sollen zur „Wiederannahme der Ungläubigkeit“ überzeugen, entgegen der populärsten Lesart der fiktionalen - vor allem fantastischen - Literatur, die in der „willentlichen Aussetzung der Ungläubigkeit" (Coleridge, 1817) besteht und auch für die utopische Liebesliteratur unbedingt scheint. ${ }^{19}$ Der desillusionierte Modellleser des ironischen Modus sollte nicht nur die Liebe der Protagonist(inn)en anzweifeln (was in der oben genannten neuen Liebeskultur nicht besonders anspruchsvoll scheint), sondern auch die Liebe in all ihren sozialen Facetten differenzieren und reflektieren können.

Die Ursprünge des ironischen Liebesschreibens lokalisiert Illouz schon bei Don Quixote (1605-1615), am Anfang der neuzeitlichen Romangeschichte. ${ }^{20}$ Ohne Zweifel könnte seine Karriere auch weiter so breit aufgefasst werden, von Cervantes über de Sade, Balzac, Flaubert, Schnitzler und Henry Miller bis in die Postmoderne. Da die vorliegende Studie jedoch nicht auf einen diachronen Vergleich unterschiedlicher Liebesromane, sondern auf eine Analyse einer spezifischen Tendenz in der aktuellen Literatur abzielt, beschränken wir uns auf die Annahme, dass der ironische Modus erst in der zweiten Hälfte des 20. und Anfang des 21. Jahrhunderts als Effekt der relativistischen Wende deutlich zum Vorschein kommt, vor allem in metafiktional aufgebauten Texten, wie etwa bei John Fowles (Die Geliebte des französischen Leutnants, 1969), A. S. Byatt (Besessen, 1990) oder Ian McEwan (Abbitte, 2001), oder in den ironischen und zugleich gesellschaftskritischen Romanen von Albert Cohen (Die Schöne des Herrn, 1968), Elfriede Jelinek (Die Liebhaberinnen, 1975) oder Michel Houellebecq (Elementarteilchen,

19 Vgl. Pearce, Romance Writing, 186.

20 Vgl. Illouz, Warum Liebe weh tut. Eine soziologische Erklärung, 350. 
1998). Wie oben bemerkt, sind vor allem kanonische Feldformationen am radikal ironischen Modus interessiert, abgesehen von einigen rein hedonistischen Tendenzen in der Unterhaltungsliteratur (wie z. B. die genannte chick-lit und ihre männliche Entsprechung lad-lit). Zu bedenken wäre auch, ob die genuin pornografische Literatur nicht im ironischen Modus zu verstehen wäre, da sie die Utopie durch maximale Intensivierung des Körperlichen aufhebt, jegliche Metaphysik ausschließt, demzufolge auch keinem Liebesprojekt Platz übriglässt. Hier kommt jedoch der Modus selbst an die Grenzen des Genres, das die Liebe und nicht den Sex als zentrales Thema behandeln sollte, ${ }^{21}$ und wenn er auch in der Pornografie wirksam ist (wahrscheinlich nur durch bloße Desemantisierung des romantischen Gefühls, da von „ironischem Porno“ kaum die Rede sein kann), kann das nicht mehr Gegenstand dieser Überlegungen sein.

Der ironische Modus ist allerdings mit dem postmodernen Schreiben nicht identisch, so wie der utopische Modus mit der idealistischen Philosophie nicht gleichgesetzt werden sollte. Die beiden verweisen zwar auf Anschauungen der gegebenen Zeit (es kann nicht anders sein), scheinen aber zugleich über enge Epochen- oder Disziplingrenzen hinauszugehen. Es wäre auch übertrieben, allen Autor(inn)en, die den ironischen Modus verwenden, unbedingt eine relativistische Auffassung der Liebe oder kritische Absichten und den Vertreter(inne)n des utopischen Modus unzeitgemäße Naivität beizumessen. Anstatt ihre Texte intentional (gemäß der Intention des Autors / der Autorin) zu erschließen, scheint eine textuelle und kontextuelle Herangehensweise angebrachter und vorsichtiger, wenn auch nur um das für den utopischen Modus charakteristische Rezeptionsschema der Gleichschaltung von intentio operis und intentio auctoris nicht zu wiederholen.

Im Folgenden werden aktuelle deutschsprachige Liebesromane (d. h. Romane, die sich als Liebesliteratur interpretieren lassen) dargestellt, die auf dem ironischen Modus aufbauen. Ausgegangen wird von Romanen, die eine unbestreitbar verfremdete, artifizielle und metafiktionale Struktur aufweisen, bis hin zu Texten, die der Darstellung der Liebesgeschichte mit ironischen Stilmitteln beizukommen versuchen.

Als besonders raffinierter, metatextueller und autofiktionaler Roman hebt sich Das Wetter vor 15 Jahren (2006) von Wolf Haas von anderen ab und bildet den ersten Teil in der Liebesromanreihe des bisher mit Krimis assoziierten Autors (hier auch Verteidigung der Missionarsstellung, 2012 und Junger Mann, 2018). Der Text, konstruiert als Interview zwischen der als „Literaturbeilage“ bezeichneten Journalistin und dem „Autor Wolf Haas“ über dessen Liebesroman, eröffnet nicht nur einen Raum für metafiktionale

21 Vgl. Lepape, Une histoire des romans d'amour, 15. 
Spiele, autofiktionale Enthüllungen und metakritische Reflexion über das literarische Schaffen an sich, sondern auch für eine Liebesgeschichte, die Gegenstand des fiktiven Romans ist, und für eine weitere, die sich allem Anschein nach zwischen den beiden Gesprächspartnern anbahnt. „Nicht nur die Satire, auch die Parodie darf alles und ihr ist es sogar möglich, den romantischen Liebescode zu retten. Vor allem durch das Distanz erzeugende Mittel der Ironie kann sie ihn neu erschaffen," ${ }^{22}$ behauptet Stefan Neuhaus in Bezug auf Haas' Roman. Vom entgegengesetzten Standpunkt aus könnte die These gewagt werden, dass eben die mehrfache Fiktionalisierung und der ironische Stil die sich weniger abspielende als gespielte oder auch verspielte Liebesgeschichte verfremden und den romantischen Code als unwiederbringlich künstlich und unglaubwürdig geworden entblößen. In dieser Hinsicht bildet Das Wetter vor 15 Jahren das Paradebeispiel des ironischen Modus, indem auf Illusionsbildung verzichtet und die ohnehin absurde Liebesgeschichte auf eine Anzahl von gekonnten Spiegelungen und formalen Verfahren reduziert wird, unter denen die Deklaration „Also ein Liebesroman ist einfach schwer zu schreiben, wenn man den Personen nicht eine gewisse Romantik lässt" ${ }^{23}$ nur als Parodie dieser zu verstehen ist.

Die genannten Verfahren - die ungewöhnliche Struktur, die Konstruiertheit von Handlung und Figuren, ausgeprägte Meta-, Para- und Intertextualität, Autofiktion, das Spiel mit Genres und der ironische Stil, deren dichte Anhäufung in Haas' Werk seine Einzigartigkeit ausmacht - finden auch Anwendung in vielen anderen Romanen der letzten Jahre. Der ebenso von Haas stammende Roman Verteidigung der Missionarsstellung (2012) scheint nicht weniger ironisch, wieder tritt der Autor als literarische Figur auf, wieder ist der Text voll von autotelischen Verfahren und der Protagonist ein Sonderling mit einer merkwürdigen Liebesgeschichte. Diesmal ereignen sich seine romantischen Abenteuer parallel zu den weltweit ausbrechenden Seuchen, was den alten Topos der Liebe als Krankheit aufruft (in der neueren Literatur vgl. Die Liebe in den Zeiten der Cholera von Gabriel García Márquez, 1985) und den Roman von Haas, gespickt von Einschüben in Klammern, die darauf hinweisen, wie die Geschichte weiter gestaltet werden sollte, umso artifizieller erscheinen lässt. Die Verliebtheit des Protagonisten dient hier einerseits der Vermehrung von paradoxalen Effekten, wie Verwechslung von Ursache und Wirkung (z. B. die zufälligen Pandemien), andererseits rechtfer-

22 Stefan Neuhaus, „Paarbildungen. Figurationen der Liebe in Gegenwartsliteratur und -film“, in Figurationen der Liebe in Geschichte und Gegenwart, Kultur und Gesellschaft, hrsg. v. Stefan Neuhaus (Würzburg: Königshausen \& Neumann, 2012), 291.

23 Wolf Haas, Das Wetter vor 15 Jahren (München: Deutscher Taschenbuch Verlag, 2014), 199. 
tigt sie das Provisorische des Romans, das ständige work in progress, z. B. die wenig glaubwürdige Länge der Dialoge: „,Diese Brücke ist sehr kurz, sie führt nur über den sehr schmalen Kanal, und wir gehen schon so lange darüber', sagte Benjamin Lee Baumgartner. ,Das ist vielleicht, weil wir verliebt sind'." ${ }^{24}$

Durch die Aufzählung von Krankheiten und Liebschaften dekonstruiert der Roman den idealistischen Liebesmythos. Aufzählen als höchst künstlerische und künstliche Figur ermöglicht eine weitgehende Ironisierung der dargestellten Geschichte - das Aufgezählte ist nicht einzigartig, nur eine Station, weder Mittel- noch Höhepunkt. Dass es trotzdem auch möglich ist, auf der Basis der Aufzählung ein Liebesideal zu beschwören, beweist Monique Schwitter in ihrem Roman Eins im Andern (2015). In zwölf Kapiteln berichtet die Erzählerin von zwölf Männern aus ihrem Leben und liefert auch Überlegungen zur unveränderlichen Natur der Liebe: „Bietet sie sich einfach in einem Mann nach dem anderen dar, offenbart sie, die eine, einzige, wahre, sich einfach nur in verschiedenen Gestalten? Hat also nicht die Liebe verschiedene Gesichter, sondern einzig der Geliebte?" ${ }^{\prime 25}$ Das utopische Anliegen des Romans wird jedoch von seiner Konstruktion selbst in Frage gestellt: Die Erzählerin hatte zwar nur fünf Lieben in ihrem Leben, da aber die beiden ersten die Namen von Petrus und Andreas tragen, scheint es unumgänglich, die Liebesgeschichte in zwölf Kapiteln weiterzuspinnen, die auf die zwölf Apostel verweisen. „Wie ich zähle, hängt davon ab, was ich erzähle," ${ }^{26}$ so räumt sie der Fiktion den Vorrang ein, ohne die der Liebesgeschichte zugrundeliegende Wahrheit zu verletzen. Oder anders: indem sie diese Wahrheit als Fiktion, nur im Rahmen des literarischen Textes verständlich und stimmig, herausstellt. Auf diese Weise scheinen sowohl freies Konfabulieren als auch Verteidigung von Grundsätzen genauso akzeptabel, was die Annahme bestätigt, dass in der Struktur des Romans Spuren des ironischen Modus zu bemerken sind, der dieses doppelbödige Spiel ermöglicht.

Das Aufzählen als Erzählfigur ist auch in zwei anderen, vollkommen unterschiedlichen Romanen zu finden. In Christoph Höhtkers Das Jahr der Frauen (2017) nimmt sich der Protagonist vor, zwölf Frauen binnen eines Jahrs zu verführen und sich auf diese Weise von Selbstmordgedanken abzulenken. In Peter Handkes Don Juan (erzählt von ihm selbst) (2004) berichtet der Protagonist von sieben Frauen, die er jeden Tag innerhalb einer Woche getroffen hat. Die beiden Don Juans, so unvereinbar sie sind, veranschaulichen die Künstlichkeit der Liebesbegegnungen in der Literatur - Höhtkers

24 Wolf Haas, Verteidigung der Missionarsstellung (München: Deutscher Taschenbuch Verlag, 2014), 28.

25 Monique Schwitter, Eins im Andern (Frankfurt am Main: Fischer, 2017), 64.

26 Schwitter, Eins im Andern, 46. 
Figur tut das aus Zynismus und Berechnung, Handkes Held aus Neigung zum Symbolischen, Mythischen, Geschlossenen. Der Unterschied zwischen dem Ironiker und dem Utopisten wird hier zwar nicht aufgehoben, mindestens aber in einer relativierenden Lesart - umgangen und mit Distanz betrachtet. So wie auch die Romane von Haas und Schwitter beweisen, dass die Liebesliteratur gerne auf deutlichen Erzählstrukturen beruht, die auch Erkenntnisstrukturen sind, und dass eine strenge Regelmäßigkeit, Vergleichen und Aufzählen verdächtig genug sind, um ein Spielfeld der subversiven Ironie zu bilden.

Handkes Beschwörung der kanonischen Figur der Liebesliteratur ${ }^{27}$ führt in den Bereich der metahistorischen Romane, wo ironische Verfahren ostentativ verwendet werden. Martin Walsers Ein liebender Mann (2008) erzählt die Geschichte von Goethes Liebe zu Ulrike von Levetzow, zitiert dabei auch seine klassischen Werke. Mehrfache ironische Spiegelungen der Biografie Goethes und der Fiktion Walsers (und vielleicht auch der Biografie Walsers) führen zu ernüchternden Erkenntnissen. Goethe und Ulrike verkleidet als Werther und Lotte bezeugen eine anfängliche Erschöpfung der Liebeskultur, die im 21. Jahrhundert zum Kampf von Selbstinszenierungen geworden ist. Einerseits liefert Walser eine durchaus ironische Diagnose der Zeit (wobei es sich mehr um die Gegenwart als um die Weimarer Klassik handelt), andererseits verteidigt er, wie gewöhnlich, den Wert der Liebe und entfaltet für ihn typische Epiphanien eines verliebten Mannes.

Ebenso doppeldeutig scheint der Roman Die Frau, für die ich den Computer erfand (2009) von Friedrich Christian Delius. Ähnlich wie bei Haas wird hier ein fiktives Interview geführt, und zwar zwischen einem unbekannten Autor (der sich nur am Anfang und am Ende äußert) und dem historischen Computererfinder Konrad Zuse. Seiner Entdeckung liegt eine merkwürdige Geschichte zugrunde, eine Geschichte der Liebe zu der bereits lange verstorbenen Ada Lovelace, der Tochter von Lord Byron. Fasziniert von einer der ersten Mathematikerinnen der Welt entwickelt der Protagonist ein Spiel von Fantasien und (Selbst)Gesprächen, in denen Ada zu seiner Begleiterin und Beschützerin wird. „Meine Phantasie hatte sie längst vorher erfunden, ich hatte sie fast fertig im Kopf ausgemalt" 28 und weiter: „, sie hat mich sofort verwandelt... Verzaubert, wenn ich das so platt sagen darf... Ich habe mich sofort verliebt, zuerst in ihren Namen verliebt“29 und weiter: „Die Frau, die

27 Interessanterweise ist er nicht der einzige unter zeitgenössischen Autoren, die ihn wiederentdeckten, vgl. Hanns-Josef Ortheils, Die Nacht des Don Juan (2000) und Robert Menasses, Don Juan de la Mancha oder Die Erziehung der Lust (2007).

28 Friedrich Christian Delius, Die Frau, für die ich den Computer erfand (Reinbek b. Hamburg: Rowohlt, 2011), 84.

29 Delius, Die Frau, für die ich den Computer erfand, 85. 
mich erfand, “30 gesteht der Protagonist und liefert so ein prägnantes Beispiel des utopischen Denkens über die Liebe - man kennt seine Liebe noch vor der Verliebtheit, Liebe verwandelt, Liebe erfindet, mehr noch: Liebe ist Ursprung jeden Fortschritts, die geheimnisvolle vitale Kraft des Lebens. Referenzen zu Goethes Faust verstärken das dezidiert idealistische Konzept dieser Liebesgeschichte, wohingegen die metafiktionale, intertextuelle, kontrafaktische und wahrscheinlich auch autofiktionale (anonymer Interviewer als Schriftsteller) Gestaltung gegen eindeutige Lesarten im Geist der Utopie wirkt. Die Anzahl der verfremdenden Verfahren und Motive, angefangen von der fantastischen Liebe zur verstorbenen und nie gekannten Frau, hinterfragen vielmehr die Glaubwürdigkeit der Geschichte, rücken sie in die Nähe von postmodernen, quasi-biografischen und metahistorischen Romanen, unter denen Die Entdeckung der Langsamkeit (1983) von Sten Nadolny und Die Vermessung der Welt (2005) von Daniel Kehlmann ein neues Genre für sich beanspruchen.

Die Behauptung, dass die neue Liebesliteratur nur fiktive oder historische Figuren bevorzugt, widerlegen unter anderem die Romane von Navid Kermani, die als autofiktional oder auch autobiografisch gelesen werden können. ${ }^{31}$ Sowohl Große Liebe (2014) als auch Sozusagen Paris (2016) stellen die Liebesgeschichte eines Schriftstellers dar, der sie später analysiert und kontextualisiert. Das intertextuelle Arsenal der Romane besteht im ersten Teil vor allem aus Referenzen zum persischen und arabischen Schrifttum des Mittelalters, im zweiten dagegen zu Klassikern der französischen Liebesliteratur, allen voran zu Proust. In diesen Rahmen fungieren die ziemlich konventionellen Erlebnisse des Protagonisten als Fallbeispiele. Wenn in Große Liebe die erste romantische Affäre eines Schülers erzählt wird, dann handelt es sich in Sozusagen Paris um Höhepunkte und Enttäuschungen einer erfahrenen Ehe. Die beiden Werke könnten die Vermutung nahelegen, dass Liebesgeschichten immer vorherigen Kulturtexten entstammen, dass die konventionellen Liebesmuster eher sekundär und Produkte medialer Erfahrung sind. Dies ist jedoch nicht der Fall. Angesichts der massenweisen Produktion von Liebesartefakten wählt Kermani nicht den relativistischen (was die Menge der intertextuellen Verweise und der ironische Titel Große Liebe nahelegen könnte ${ }^{32}$ ), sondern den idealistischen Diskurs: „Was wir als trivial wahrnehmen, weil es industriell kopiert wird - reflektiert es nicht eine Grunderfahrung, die die meisten als Jugendliche gemacht?“33

30 Delius, Die Frau, für die ich den Computer erfand, 90.

$31 \mathrm{Ob}$ das auch vom Autor intendiert ist, bleibt unklar, auf jeden Fall werden seine Romane eben in diesem Modus wahrgenommen.

32 Vgl. auch den Roman Die große Liebe (2003) von Hanns-Josef Ortheil, der allerdings - was bei diesem Autor nicht verwundern soll - die große Liebe tatsächlich legitimieren will.

33 Navid Kermani, Große Liebe (München: Hanser, 2014), 31. 
Kermanis Romane führen jedoch in eine dezidiert ironische Richtung Konstruktion, Rekonstruktion und Dekonstruktion der einmal erlebten Liebesgeschichte. Der Begriff "Geschichte" setzt in der relativistischen Auffassung (u. a. Hayden White) einen Modus des Erzählens voraus, eine Künstlichkeit der Darstellung, die in Auswahl, Stilisierung und Zusammensetzung der vorhandenen Elemente besteht. Der Zwang, eine Kohärenz in die erzählte Liebesgeschichte einzubringen, eine für alle verständliche Logik, die es jedoch auch nicht unbedingt gab oder geben muss, fiktionalisiert den erzählten Gegenstand. Es scheint, dass diese Konstruiertheit im ironischen Modus gerne zur Schau getragen wird, mehr noch: sie kann auch im utopischen Modus nicht unbeachtet bleiben. Der bereits zum Klassiker gewordene Roman von Peter Stamm Agnes (1998) berichtet vom Schicksal eines Paares, das beginnt, seine gemeinsame Liebesgeschichte aufzuschreiben. Der Roman Stamms und der parallel niedergeschriebene Roman des Erzählers enthüllen die Geschichte als Konstrukt und Effekt unzähliger Experimente und Kompromisse. Noch deutlicher kommt dieser Sachverhalt in Brigitte Kronauers Der Scheik von Aachen (2016) zum Vorschein. Hier verliert die Protagonistin Anita ihren Partner und beginnt eine neue Affäre, während sie sich inzwischen mit den tragischen Familiengeheimnissen auseinandersetzt. Das dramatische Fundament der Erzählung wird jedoch von regelmäßigen metafiktionalen Einschüben unterspült, die die Protagonistin in ihrer Rolle als literarische Figur in den Vordergrund stellen: „Zu wem spricht sie? Zunächst zu sich, dann zu Ihnen, jawohl, zu Ihnen, auch wenn Sie für Anita noch viel fiktiver sind als umgekehrt." ${ }^{34}$ In diesem Geflecht von Fiktionen erscheinen auch die Worte des Protagonisten Marzahn beinahe als Programm: „Die Liebe, Sie wissen es in Wirklichkeit so gut wie ich, kommt immer aus den Gaukeleien der Literatur“35, auch wenn die hier dargestellte (vielfache) Liebesgeschichte glücklich endet und dem Antiquitätenhändler Marzahn nur die klassische Funktion des extravaganten Nörglers zufällt.

Radikalisiert werden diese verfremdenden Signale in Olga Flors Roman Klartraum (2017). Die Liebesgeschichte der Protagonistin P und des Antagonisten A (so die Namen der Figuren) kulminiert in einer Ansprache an die Leserschaft, die Liebesromane kauft und genießt. Das Schreiben der Liebesliteratur wird zur reinen Dienstleistung, die den Wünschen der Lesenden gerecht zu werden versucht: „Rundherum versinken Staaten im Chaos, Boote im Meer, das entzieht sich unserem Zugriff. [...] Dann erfüllen wir lieber Ihren Eskapismusanspruch, dafür zahlen Sie schließlich. “36 Falls das Publikum

34 Brigitte Kronauer, Der Scheik von Aachen (München: Deutscher Taschenbuch Verlag, 2019), 9.

35 Kronauer, Der Scheik von Aachen, 236.

36 Olga Flor, Klartraum (Salzburg: Jung und Jung, 2017), 235. 
genug von romantischen Abenteuern hätte, wäre auch „eine Leibesge-

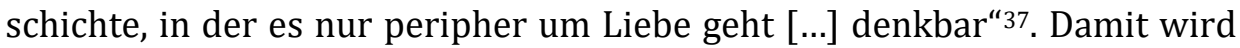
auch eine ironische Selbstanspielung zustande gebracht, da der Roman Flors - voll von essayistischen Passagen und Verfremdungseffekten - sich möglichst fern von der üblichen Vorstellung vom Genre Liebesroman situiert und offensichtliche romantische und erotische „Momente“ meidet.

Diese selbstreflexive, extrem ironische Sprache teilt Flor mit anderen Autorinnen, die für die Darstellung der Geschlechterverhältnisse äußerst unkonventionelle, illusionsbrechende Mittel wählen. Elfriede Jelineks Anklage der männlichen Dominanz, der körperlichen Instrumentalisierung von Frauen und der popkulturellen romantischen Mythen findet im Roman Gier (2000) ihren wahrscheinlich prägnantesten Ausdruck. Bereits der Untertitel Ein Unterhaltungsroman verweist ironisch auf die herkömmliche Klassifizierung von Liebesgeschichten und ist ein eindeutiges Signal, dass hier vom Unterhaltungsroman keine Rede sein kann. Die für Jelinek typischen selbstreflexiven Monologe, Sprachspiele, sarkastische Darstellung von Gewalt und Sex sowie dichte intertextuelle Verweise machen jegliche Identifikation unmöglich und rücken diesen „Antiroman“ 38 weg von der Sparte „Unterhaltung" in den Bereich der Diskurskritik.

Hier lassen sich auch die Romane von Sibylle Berg verorten, in dem besprochenen Kontext vor allem Der Tag, als meine Frau einen Mann fand (2015), ein dialogischer Bericht von der Niederlage fester Beziehungen, Ziellosigkeit der sexuellen Ausschweifung und Monotonie des Liebeslebens am Anfang des 21. Jahrhunderts. „Ich habe mich [...] gegen den Sex entschieden und für die Liebe. Sieg der Vernunft über die Begierde. Heute haben wir Sex, wenn ich morgens hart bin, weil die Blase auf meine Prostata drückt,"“39 gesteht der infantile Protagonist Rasmus, ein gescheiterter Theatermacher, der gegen die Ereignislosigkeit seines Daseins nur falsche Entscheidungen trifft. Er und seine Frau Chloe leben in einer Welt von Ängsten, Komplexen und Unsicherheit, die in Bergs ironischer Darstellung Züge eines grotesken Theaters annimmt.

Von dieser drückenden Unsicherheit sind auch Figuren von Marlene Streeruwitz gepeinigt, die in der Auseinandersetzung mit ihrer (hauptsächlich männlichen oder durch männliche Wünsche kreierten) Umgebung verletzt werden, metaphorisch oder auch buchstäblich wie die vergewaltigten

37 Flor, Klartraum.

38 Vgl. Helen Finch, „Elfriede Jelinek’s Gier (Greed)“, in The Novel in German since 1990, hrsg. v. Stuart Taberner (Cambridge: Cambridge University Press, 2011), 153, https://doi.org/ 10.1017/CB09780511667558.

39 Sibylle Berg, Der Tag, als meine Frau einen Mann fand (München: Hanser, 2015), 24. 
Protagonistinnen in Jessica, 30 (2004) und Die Schmerzmacherin (2008). Streeruwitz, die früher in Lisa's Liebe (1997) triviale Liebesromane direkt parodiert hat, wird in ihrem reiferen Schaffen einfühlsam, begleitet ihre Protagonistinnen in ihre Intimsphäre, beobachtet ihre alternden Körper, und wenn sie schon "Liebesromane" schreibt, dann sind das Zeugnisse vom Scheitern des sensiblen und einsamen Individuums angesichts der überindividuellen Illusion der Liebe.

Ohne das Materielle ist die Liebesliteratur kaum möglich, auch die angeblich „platonische“, in der der Körper nur ein Träger von Stimmen und Gedanken oder aber eine Bedrohung ist, die bei Sibylle Berg sehr plausibel versprachlicht wird: „Er ist in sexueller Stimmung und wird [...] sein Glied, dieses Körperteil, das Unruhe in unsere Liebe bringt, in mich stecken." ${ }^{40}$ Auch Romane wie Daniel Glattauers Gut gegen Nordwind (2006), die ohne Erzähler, Monologe, innere Welt der Protagonist(inn)en und auch ohne Körper auskommen, weil sie nur aus E-Mails bestehen, scheinen vom Körperlichen „bedroht“. Besonders im voyeuristischen 21. Jahrhundert können sie ohne den Gedanken an Körper nicht gelesen werden. In Corinna T. Sievers' Die Halbwertszeit der Liebe (2016) betrachtet die Protagonistin ihre Gefühle und ihren in perversen Spielen agierenden Körper mit wissenschaftlicher Gleichgültigkeit, im nächsten Roman der Autorin, Vor der Flut (2019), ist das Wort Liebe fast ausschließlich gleichbedeutend mit Begehren und Sex. Die nymphomane Zahnärztin, die hier erotische Abenteuer sammelt und ihren Ehemann alleine sterben lässt, desillusioniert die Liebe als Erzählung von Treue, Verbundenheit und Empathie, was von der nüchternen, gefühllosen Sprache nur verstärkt wird. Die Romane von Sievers könnten als Pendant zu enthusiastischen Promiskuitätsnarrationen wie etwa Charlotte Roches Feuchtgebiete (2008) dienen, da sie das gleiche Phänomen ansprechen - die Fetischisierung des Körpers und der Körperreaktionen, allerdings von zwei unterschiedlichen Standpunkten aus. Der Roman von Roche ist bejahend, ihre Protagonistin benötigt keine Selbstbestätigung durch Sex, bei Sievers dagegen heißt es: „solange ich ficke, bin ich nicht tot, ${ }^{41}$ was (in diesem sich immer selbst erklärenden Roman) psychoanalytisch als „Bevorzugung des Lebenswunsches gegenüber dem Todestrieb“42 ausgelegt wird.

Liebesliteratur ohne Metaphysik? Liebesromane als intellektuelle Pornografie? Leibesliteratur statt Liebesliteratur (siehe Olga Flor oben)? Wenn schon, dann nicht mehr im Zeichen der Ironie. Die Körper bei Sievers sind

\footnotetext{
40 Berg, Der Tag, als meine Frau einen Mann fand, 19.

41 Corinna T. Sievers, Vor der Flut (Frankfurt am Main: Frankfurter Verlagsanstalt, 2019), 124, E-Book.

42 Sievers, Vor der Flut, 123-124.
} 
relativ neue, in ihren Lebensfunktionen unbeteiligt beobachtete, „mit schockierender Nüchternheit und so sachlich, als gelte es, [sie] aus dem pornographischen Phantasma, aus der Verwertung der Lust, zu erlösen, “43 Körper im postpornografischen Modus. Sievers' Ironie ist elementar, wirkt als sprachlicher Witz, den Kern ihrer Romane bildet aber das perfekt materielle, unstilisierte, uneigennützige (weil keine Erregung auslösende, keine Effekte und Profite erwartende) Dasein des Körpers, das weder dem theologischen Dispositiv noch der pornografischen Gewalt untergeordnet ist. ${ }^{44}$ Hier stößt der ironische Modus an seine Grenze.

Die These Thomas Hettches, ein neuer Modus der Körperdarstellung die „neue Keuschheit der Pornografie“ - gewinne zurzeit an Bedeutung, steht im Einklang mit dem mehrmals konstatierten und bereits zum Gemeinplatz der Forschung gewordenen Ende der Ironie in der deutschsprachigen Literatur. ${ }^{45}$ Dass die Ironie sich allerdings nicht in popliterarischen Stilisierungen und Spielen erschöpft und besonders gerne im neuesten Liebesroman als Instrument der Relativierung eingesetzt wird, beweist nicht nur die Beständigkeit des subversiven Schreibens über Liebe, das bereits zum kanonischen Repertoire der Postmoderne gehört, sondern auch die Wirksamkeit der größtenteils retrotopischen Liebesliteratur der letzten Jahrzehnte, die diese subversiven Reaktionen hervorruft. Den beiden Phänomenen liegt jedoch eines zugrunde: die Überzeugung, dass Liebe und Beziehungen in jeder Hinsicht konfus und ungewiss und dass „Nichtwahl“ und „Entlieben“ zu ihren Konstanten geworden sind. ${ }^{46}$ Die Dialektik von Utopie und Ironie, Eskapismus und Subversion, Rettungsfantasie und Desillusionierung erweist sich somit als das Janusgesicht der gegenwärtigen Liebeskultur.

\section{References}

Berg, Sibylle. Der Tag, als meine Frau einen Mann fand. München: Hanser, 2015.

Bourdieu, Pierre. Die Regeln der Kunst. Genese und Struktur des literarischen Feldes. Tranlated by Bernd Schwibs, and Achim Russer. Frankfurt am Main: Suhrkamp, 1999.

43 Thomas Hettche, „Die neue Keuschheit der Pornographie“, Frankfurter Allgemeine Zeitung, Nr. 17 (2003): 38.

44 Vgl. Byung-Chul Han, Transparenzgesellschaft (Berlin: Matthes \& Seitz, 2012), 36-38, E-Book.

45 Vgl. Christoph Rauen, Pop und Ironie. Popdiskurs und Popliteratur um 1980 und 2000 (Berlin - New York: De Gruyter, 2010), 2-3.

46 Vgl. Eva Illouz, The End of Love. A Sociology of Negative Relations (New York: Oxford University Press, 2019), 3-25, 73-97, E-Book. 
Delius, Friedrich Christian. Die Frau, für die ich den Computer erfand. Reinbek b. Hamburg: Rowohlt, 2011.

Finch, Helen. "Elfriede Jelinek's Gier (Greed)." In The Novel in German since 1990, edited by Stuart Taberner, 151-164. Cambridge: Cambridge University Press, 2011, https://doi.org/10.1017/CB09780511667558.

Flor, Olga. Klartraum. Salzburg: Jung und Jung, 2017.

Haas, Wolf. Das Wetter vor 15 Jahren. München: Deutscher Taschenbuch Verlag, 2014.

Haas, Wolf. Verteidigung der Missionarsstellung. München: Deutscher Taschenbuch Verlag, 2014.

Han, Byung-Chul. Agonie des Eros. Berlin: Matthes \& Seitz, 2017.

Han, Byung-Chul. Transparenzgesellschaft. Berlin: Matthes \& Seitz, 2012. E-Book.

Hettche, Thomas. "Die neue Keuschheit der Pornographie." Frankfurter Allgemeine Zeitung, no. 17 (2003): 38.

Illouz, Eva. Der Konsum der Romantik. Liebe und die kulturellen Widersprüche des Kapitalismus. Translated by Andreas Wirthensohn. Frankfurt am Main: Suhrkamp, 2007.

Illouz, Eva. The End of Love. A Sociology of Negative Relations. New York: Oxford University Press, 2019. E-Book.

Illouz, Eva. Warum Liebe weh tut. Eine soziologische Erklärung. Translated by Michael Adrian. Berlin: Suhrkamp, 2011.

Kermani, Navid. Große Liebe. München: Hanser, 2014.

Kronauer, Brigitte. Der Scheik von Aachen. München: Deutscher Taschenbuch Verlag, 2019.

Lachmann, Renate. Erzählte Phantastik. Zu Phantasiegeschichte und Semantik phantastischer Texte. Frankfurt am Main: Suhrkamp, 2002.

Lepape, Pierre. Une histoire des romans d'amour. Paris: Seuil, 2011.

Luhmann, Niklas. Liebe als Passion. Zur Codierung von Intimität. Frankfurt am Main: Suhrkamp, 2015.

Morikawa, Takemitsu. "Gibt es ein Weltfunktionssystem für Intimbeziehungen? Jenseits des Spannungsverhältnisses von Kulturalismus und Westzentrismus." In Liebesgeschichte(n). Identität und Diversität vom 18. bis zum 21. Jahrhundert, edited by Frank Becker, and Elke Reinhardt-Becker, 331-360. Frankfurt am Main: Campus, 2019.

Neuhaus, Stefan. "Paarbildungen. Figurationen der Liebe in Gegenwartsliteratur und -film." In Figurationen der Liebe in Geschichte und Gegenwart, Kultur und Gesellschaft, edited by Stefan Neuhaus, 273-292. Würzburg: Königshausen \& Neumann, 2012.

Pearce, Lynne. Romance Writing. Cambridge: Polity Press, 2007.

Rauen, Christoph. Pop und Ironie. Popdiskurs und Popliteratur um 1980 und 2000. Berlin - New York: De Gruyter, 2010. 
Rorty, Richard. Contingency, irony, and solidarity. Cambridge: Cambridge University Press, 1993, https://doi.org/10.1017/CB09780511804397. Schwitter, Monique. Eins im Andern. Frankfurt am Main: Fischer, 2017. Sievers, Corinna T. Vor der Flut. Frankfurt am Main: Frankfurter Verlagsanstalt, 2019. E-Book.

Szczepaniak, Monika. "Coole Romanze als literarisches Programm." In Liebesgeschichte(n). Identität und Diversität vom 18. bis zum 21. Jahrhundert, edited by Frank Becker, and Elke Reinhardt-Becker, 151-169. Frankfurt am Main: Campus, 2019.

Tommek, Heribert. Der lange Weg in die Gegenwartsliteratur. Studien zur Geschichte des literarischen Feldes in Deutschland von 1960 bis 2000. Berlin - New York: De Gruyter, 2015.

\title{
Der ironische Modus im aktuellen Liebesroman
}

\begin{abstract}
Der ironische Modus ist ein Komplex von literarischen, vor allem strukturellen, metafiktionalen, intertextuellen, verfremdenden und illusionsbrechenden Verfahren, die im aktuellen Liebesroman eingesetzt werden. Dem liegt ein relativistisches Verständnis der Liebe als kulturelles Konstrukt zugrunde. In diesem Beitrag wird der ironische Modus dem utopischen entgegengesetzt sowie nach seiner Wirksamkeit in der neuesten deutschsprachigen Literatur gefragt. Angesprochen werden Liebesromane von Wolf Haas, Monique Schwitter, Christoph Höhtker, Peter Handke, Martin Walser, Friedrich Christian Delius, Navid Kermani, Brigitte Kronauer, Olga Flor, Elfriede Jelinek, Sibylle Berg, Marlene Streeruwitz und Corinna T. Sievers.
\end{abstract}

Schlüsselwörter: Liebesroman, Liebe, Ironie, deutschsprachige Gegenwartsliteratur.

\section{Tryb ironiczny we współczesnej powieści miłosnej}

\begin{abstract}
Abstrakt: Tryb ironiczny stanowi zespół literackich, przede wszystkim strukturalnych, autotelicznych, intertekstualnych, dystansujących i znoszących iluzję chwytów, które znajdują zastosowanie we współczesnych powieściach miłosnych. U podstaw tego trybu leży relatywistyczne rozumienie miłości jako kulturowego konstruktu. Artykuł proponuje analizę trybu ironicznego w opozycji do utopijnemu trybu pisania o miłości. Jej podstawę tworzą powieści Wolfa Haasa, Monique Schwitter, Christopha Höhtkera, Petera Handkego, Martina Walsera, Friedricha Christiana Deliusa, Navida Kermaniego, Brigitte Kronauer, Olgi Flor, Elfriede Jelinek, Sibylle Berg, Marlene Streeruwitz oraz Corinny T. Sievers.
\end{abstract}

Słowa kluczowe: powieść miłosna, miłość, ironia, współczesna literatura niemieckojęzyczna. 